\title{
USP22 promotes melanoma and BRAF inhibitor resistance via YAP stabilization
}

\author{
YING WEI $^{1 *}$, ZIYUN JIANG $^{2 *}$ and JIANFENG LU ${ }^{1}$ \\ ${ }^{1}$ Department of Plastic and Reconstructive Surgery, Changxing People's Hospital, Huzhou, Zhejiang 313100; \\ ${ }^{2}$ Research Department, Shanghai Zhuole Biotechnology Center, Shanghai 201499, P.R. China
}

Received July 30, 2020; Accepted February 3, 2021

DOI: $10.3892 / \mathrm{ol} .2021 .12655$

\begin{abstract}
Yes-associated protein (YAP) is a conserved transcriptional coactivator that plays key roles in controlling organ size, tumorigenesis and drug resistance. Emerging evidence shows that YAP is overexpressed and associated with resistance to BRAF inhibitor treatment in melanoma. However, the mechanism accounting for YAP-overexpression in melanoma is largely unknown. The present study characterized ubiquitin-specific peptidase 22 (USP22) as a deubiquitinase controlling YAP abundance and biological functions in melanoma. Using western blotting and immunohistochemical staining, it was found that the expression of USP22 and YAP was associated in melanoma cell lines and patient samples. Moreover, USP22 interacted with and deubiquitinated YAP to prevent YAP turnover. Depletion of USP22 decreased YAP expression, which in turn suppressed cell proliferation and tumorigenesis. Furthermore, overexpression of USP22 conferred vemurafenib resistance in a YAP-dependent manner. Overall, the present study revealed the important role of the USP22/YAP axis in melanoma and BRAF inhibitor resistance, and provides a rationale to target USP22/YAP for melanoma treatment.
\end{abstract}

\section{Introduction}

Melanoma is the most serious type of skin cancer, accounting for $\sim 70 \%$ of skin cancer-associated deaths in the United States in $2018(1,2)$. The incidence of melanoma has risen rapidly since 1975 , and 95,710 newly diagnosed cases were predicted for 2020 (3). With the development of promising new treatments, particularly targeted therapies and immunotherapy,

Correspondence to: Dr Jianfeng Lu, Department of Plastic and Reconstructive Surgery, Changxing People's Hospital, 66 Taihu Middle Road, Changxing, Huzhou, Zhejiang 313100, P.R. China E-mail: lujianfeng1203@163.com

${ }^{*}$ Contributed equally

Key words: yes-associated protein, ubiquitin-specific peptidase 22, melanoma, tumorigenesis, BRAF inhibitor, resistance among all patients with melanoma after diagnosis, the 5-year survival rate of melanoma has increased to $92 \%$ in the USA in 2019 (3-7). However, since up to 50\% of patients with melanoma do not respond to or acquire resistance to these therapies $(8,9)$, the overall mortality rate has not markedly decreased. Melanoma-associated death decreased by $\sim 6 \%$ in the USA from 2013 to 2017 (3). Therefore, it is important to investigate the molecular mechanisms to identify new targets/strategies for the improved treatment of melanoma.

Most melanomas arise from recurrent somatic mutations, which lead to the dysregulation of oncogenic pathways that regulate cell proliferation, apoptosis and invasion (10-12). The most frequently mutated pathway in melanoma is the MAP kinase (MAPK) signaling pathway, the mutation of which occurs in $\sim 70 \%$ of all melanomas and results in constitutive activation of MAPK signaling. Notably, $\sim 50 \%$ of melanomas harbor $B R A F$ oncogenic mutations, with $\mathrm{V} 600 \mathrm{E}$ being the most common $(13,14)$. Therefore, targeting the BRAF-V600E mutation has attracted notable research attention. Several inhibitors antagonizing this mutation have been developed, two of which (vemurafenib and dabrafenib) have been approved by the Food and Drug Association for treatment of non-resectable BRAF-V600E/K mutant melanoma (15). Although the short-term response is promising, most patients with melanoma acquire resistance to these BRAF inhibitors and progress to more aggressive disease through various mechanisms, such as other genetic alterations restoring the MAPK pathway or activating the PI3K/Akt signaling pathway (16). However, $\sim 40 \%$ of patients develop resistance with unknown causes (17).

Yes-associated protein 1 (YAP) is an evolutionarily conserved transcriptional coactivator that functions as a key regulator of organ development, cancer progression and therapeutic resistance (18). YAP governs cell proliferation and apoptosis by controlling transcriptional programs through interacting with transcription factors, such as TEAD and $\beta$-catenin (19). In support of YAP as an oncoprotein, overexpression of YAP has been associated with a variety of human cancer types, including lung and breast cancer $(20,21)$. Previous studies have shown that YAP promotes melanoma cell proliferation, invasion and resistance to BRAF/MEK inhibitors (22-25). Consistently, patients with melanoma with high expression of YAP display less responsiveness to RAF/MEK inhibitors (25). However, the molecular 
mechanism for the overexpression of YAP in melanoma is largely unknown.

YAP is a major downstream effector of the Hippo pathway, which is composed of a kinase cascade containing MAP4K, MST1/2 and LATS1/2. In response to the changes in microenvironment, such as cell-cell adhesions and mechanotransduction, activated LATS1/2 kinases phosphorylate YAP at multiple Ser/Thr residues, leading to YAP cytoplasmic translocation (26). Other post-translational modifications also play a critical role in regulating YAP subcellular localization and transcriptional activity (27). Notably, ubiquitination-mediated proteolysis is a key mechanism controlling YAP protein levels. Several E3 ubiquitin ligases of YAP have been identified. $\beta$-transducin repeats-containing protein and F-box/WD repeat-containing protein 7 promote YAP ubiquitination and degradation in a phosphorylation-dependent manner $(28,29)$. Notably, SKP2-mediated K63-linked poly-ubiquitination of YAP is a non-proteolytic signal, which enhances YAP nuclear accumulation independent of the Hippo pathway (30). Similar to phosphorylation, ubiquitination can be reversed by deubiquitinating enzymes (DUBs), the dysregulation of which contributes to aberrant protein expression. Several DUBs have been identified to regulate YAP cytoplasmic/nuclear translocation and transcriptional activity, such as OTUD1 and YOD1 (27); however, the DUBs that directly control YAP protein stability in melanoma remain to be identified.

The current study aimed to characterize ubiquitin specific peptidase 22 (USP22) as a DUB controlling YAP abundance and biological functions in melanoma.

\section{Materials and methods}

Melanoma specimens and immunohistochemistry (IHC) analysis. The patient samples were collected by fixing into formalin within 15-30 min after surgical resection between January 2015 and December 2018 under the protocol approved by the Institutional Research Ethics Committee at Changxing People's Hospital (Huzhou, China). The median age of patients was 60 years (range, 15-85 years), and $56.7 \%$ of patients were male. All patients with resectable melanoma were included. Patients were fully informed, and written consent was obtained from all patients and/or their guardians before sample collection. In total, 90 melanoma tissue samples were fixed with $10 \%$ formalin for $24 \mathrm{~h}$ at room temperature and embedded in paraffin. The sections were cut into 5- $\mu \mathrm{m}$-thick sections and used for IHC staining with anti-USP22 antibody (1:500; cat. no. ab195289; Abcam) and YAP antibody (1:500; cat. no. 14074; Cell Signaling Technology, Inc.). IHC analysis of YAP and USP22 expression was based on visual inspection by two members of our group of staining intensity, which is commonly used by pathologists in the research field. To provide unbiased analysis, the two researchers independently scored the samples as low, medium and high using representative images as references. The association between USP22 and YAP was analyzed using the $\chi^{2}$ test. All procedures performed in the present study involving human participants were approved and in accordance with the ethical standards of the institutional and/or national research committee, and in accordance with the 1964 Helsinki declaration and its later amendments or comparable ethical standards.
Cell culture and reagents. Normal human epidermal melanocytes (NHEM; cat. no. PCS-200-013), all melanoma cell lines (SK-MEL-3, SK-MEL-28, A375, A2058 and G361) and 293T cells were obtained from the American Type Culture Collection (ATCC) and cultured according to the suppliers' instructions. The Dermal Cell Basal Medium (cat. no. PCS-200-030), McCoy's 5a Medium (cat. no. 30-2007) was used for PCS-200-013, SK-MEL-3 and G361 cells, Eagle's Minimum Essential Medium (cat. no. 30-2003) was used for SK-MEL-28 cells, and Dulbecco's Modified Eagle's Medium (DMEM; cat. no. 30-2002) was used for A375, A2058 and 293T cells. All media were purchased from ATCC. FBS was purchased from Thermo Fisher Scientific, Inc. (cat. no. 10438026), and $10 \%$ FBS was added to the medium for all cells. The cells were maintained in $5 \% \mathrm{CO}_{2}$ incubator at $37^{\circ} \mathrm{C}$. BRAF-V600E inhibitor vemurafenib (cat. no. S1267) and proteasome inhibitor MG-132 (cat. no. S2619) were purchased from Selleck Chemicals.

Cell transfection. Lipofectamine 3000 was used for transfection following the manufacturer's instructions (cat. no. L3000008; Invitrogen; Thermo Fisher Scientific, Inc.). Lentiviral packaging and infection were carried out as previously described (31). Briefly, 293T cells were transfected with $6 \mu \mathrm{g}$ lentiviral vector [short hairpin (sh)GFP (shRNA targeting GFP used as negative control), shYAP or shUSP22] and second generation packaging plasmids, $2 \mu \mathrm{g}$ psPAX2 (cat. no. 12260) and $4 \mu \mathrm{g}$ pMD2.G (cat. no. 12259) (both Addgene, Inc.) using Lipofectamine 3000 . After $18 \mathrm{~h}$ of transfection, DMEM was replaced with fresh DMEM medium. Viruses were collected at 48 and $72 \mathrm{~h}$ and then filtered with a $0.45-\mu \mathrm{m}$ PES filter. The virus titer was measured using the Lenti-X GoStix Plus kit (cat. no. 631280; Takara Bio, Inc.) following the manufacturer's instructions. Targeted cells A375 and A2058 were infected with the virus at MOI 5.0 and selected with $1 \mu \mathrm{g} / \mathrm{ml}$ puromycin for 3 days in a $5 \% \mathrm{CO}_{2}$ incubator at $37^{\circ} \mathrm{C}$ to eliminate the non-infected cells before harvesting.

Plasmids. Flag-USP22 (OHu25420; used for ectopic expression of USP22 in A375 and A2058 cells), HA-YAP (OHu15043; customized vector pcDNA3.1+N-HA; used for ectopic expression of YAP in A375 and A2058 cells) and Myc-Ub (OHu28056; customized vector pcDNA3.1+N-Myc; used for ectopic expression of ubiquitin in A375 cells for the in vivo ubiquitination assay) were purchased from GenScript. pcDNA3.1 (cat. no. V79020; Thermo Fisher Scientific, Inc.) was used as an empty vector (EV) as a negative control. The Flag-USP22-C185S mutation was generated using the QuikChange XL site-directed mutagenesis kit (cat. no. 200521; Agilent Technologies, Inc.). shYAP1-1\# (cat. no. 42540) was purchased from Addgene, Inc. shUSP22-1\# (cat. no. TRCN0000291124) and shUSP22-2\# (cat. no. TRCN0000296867) were purchased from Sigma-Aldrich; Merck KGaA. To ectopically express pcDNA 3.1 (EV), YAP or USP22, A375 and A2058 cells were transfected with the plasmids pcDNA 3.1, HA-YAP or Flag-USP22 (5 $\mu \mathrm{g}$ each), respectively, using Lipofectamine 3000 at $37^{\circ} \mathrm{C}$ for $24 \mathrm{~h}$. After $36 \mathrm{~h}$ of transfection, cells were used for subsequent experiments. 
Western blotting and immunoprecipitation (IP). All cells used for western blotting were lysed with 1X RIPA buffer (cat. no. 9806; Cell Signaling Technology, Inc.) containing 1X protease inhibitor cocktail (cat. no. 78429; Thermo Fisher Scientific, Inc.) and incubated for $15 \mathrm{~min}$ with rotation at $4^{\circ} \mathrm{C}$. After centrifugation at $15,871 \mathrm{x}$ g for $10 \mathrm{~min}$ at $4^{\circ} \mathrm{C}$, the supernatant was collected and the protein concentration was measured using the BCA method. The samples were boiled at $95^{\circ} \mathrm{C}$ for $10 \mathrm{~min}$. In total, $25-50 \mu \mathrm{g}$ proteins in each lane were separated using a $10 \%$ gel for SDS-PAGE and transferred to a PVDF membrane, followed by blocking with $5 \%$ skimmed milk in $1 \mathrm{X}$ TBS containing $0.1 \%$ Tween-20 (TBST) buffer (cat. no. BUF028; Bio-Rad Laboratories, Inc.) at room temperature for $30 \mathrm{~min}$. Next, the membrane was incubated with primary antibodies at $4^{\circ} \mathrm{C}$ overnight. After washing three times with $1 \mathrm{X}$ TBST buffer, the membrane was incubated with secondary antibody at room temperature for $1 \mathrm{~h}$, followed by washing three times. All the primary antibodies were used at a dilution of 1:1,000 in $5 \%$ skimmed milk, and the secondary antibodies were used at a dilution of 1:3,000 in 5\% skimmed milk. The anti-YAP (cat. no. 14074), anti-GAPDH (cat. no. 5174), anti-ubiquitin (cat. no. 43124), anti-Myc-tag (cat. no. 2272), anti-cleaved PARP (cPARP; cat. no. 5625), anti-HA (cat. no. 3724), anti-Flag (cat. no. 14793) and anti-rabbit secondary antibody linked with HRP (cat. no. 7074) were purchased from Cell Signaling Technology, Inc. The anti-USP22 antibody (cat. no. sc-390585) was purchased from Santa Cruz Biotechnology, Inc. The western blot images were developed using the chemiluminescence detection kit (cat. no. WBKLS0500; EMD Millipore), the ChemiDoc Imaging system (Bio-Rad Laboratories, Inc.) and the Image Lab 6.1 software (Bio-Rad Laboratories, Inc.). For immunoprecipitation, 1,000 $\mu \mathrm{g}$ whole cell lysates were incubated with $2 \mu \mathrm{g}$ primary antibodies, including anti-USP22 (cat. no. ab195289; Abcam), anti-YAP (cat. no. 14074; Cell Signaling Technology, Inc.) or anti-HA (cat. no. 3724; Cell Signaling Technology, Inc.), for $6 \mathrm{~h}$ and then incubated with $20 \mu \mathrm{l}$ protein A agarose (cat. no. 20333; Thermo Fisher Scientific, Inc.) for $1 \mathrm{~h}$. The immunoprecipitants were centrifuged at $15,871 \mathrm{x} \mathrm{g}$ for $1 \mathrm{~min}$ at $4^{\circ} \mathrm{C}$ and then washed with RIPA buffer (Cell Signaling Technology, Inc.) three times, followed by boiling at $95^{\circ} \mathrm{C}$ for $10 \mathrm{~min}$ and finally western blot analysis performed as aforementioned.

Cycloheximide-chase assay. Cells were plated in a 60-mm cell culture dish at $60 \%$ confluence for $24 \mathrm{~h}$ and then treated with $100 \mu \mathrm{g} / \mathrm{ml}$ cycloheximide (cat. no. S7418; Selleck Chemicals) for $0,2,4,6$ or $8 \mathrm{~h}$ before harvesting for western blot analysis performed as aforementioned.

In vivo ubiquitination analysis. A375 cells transfected with Myc-Ub, Flag-USP22 and/or HA-YAP were treated with $10 \mu \mathrm{M}$ MG-132 for $12 \mathrm{~h}$ before harvesting with $1 \mathrm{X}$ cell lysis buffer (Cell Signaling Technology, Inc.) and incubating for $15 \mathrm{~min}$ with rotation at $4^{\circ} \mathrm{C}$. After centrifugation at $15,871 \mathrm{x} \mathrm{g}$ for $10 \mathrm{~min}$ at $4^{\circ} \mathrm{C}, 1,000 \mu \mathrm{g}$ supernatant was incubated with $2 \mu \mathrm{g}$ primary antibodies, including anti-YAP (cat. no. 14074; Cell Signaling Technology, Inc.) or anti-HA (cat. no. 3724; Cell Signaling Technology, Inc.), for $4 \mathrm{~h}$, followed by addition of $20 \mu 1$ protein A agarose (cat. no. 20333; Thermo Fisher
Scientific, Inc.) for $1 \mathrm{~h}$ or incubation with $20 \mu \mathrm{l}$ anti-HA agarose (cat. no. 26181; Thermo Fisher Scientific, Inc.) for $5 \mathrm{~h}$ at $4^{\circ} \mathrm{C}$ with rotation. The immunoprecipitants were washed three times with $1 \mathrm{X}$ cell lysis buffer. The samples were boiled at $95^{\circ} \mathrm{C}$ for $10 \mathrm{~min}$. Ubiquitinated YAP protein was detected using anti-ubiquitin or anti-Myc-tag and western blot analysis as aforementioned.

Reverse transcription-quantitative (RT-qPCR). Total RNA was extracted from A375 cells transfected with shUSP22 and/or shYAP using the RNeasy mini kit following the manufacturer's instructions (cat. no. 74104; Qiagen, Inc.). In total, $1 \mu \mathrm{g}$ RNA was used for cDNA synthesis using the iScript ${ }^{\mathrm{TM}}$ Reverse Transcription Supermix according to the manufacturer's instructions (cat. no. 1708841; Bio-Rad Laboratories, Inc.). The mRNA levels were examined by SYBR Green Supermix (cat. no. 1725270; Bio-Rad Laboratories, Inc.) using the following thermocycling conditions: $95^{\circ} \mathrm{C}$ for $30 \mathrm{sec}$, then 40 cycles of $95^{\circ} \mathrm{C}$ for $10 \mathrm{sec}$ and $60^{\circ} \mathrm{C}$ for $30 \mathrm{sec}$. GAPDH was used as an internal control. The relative mRNA levels were quantified using the $2^{-\Delta \Delta \mathrm{Cq}}$ method as described previously (32). The primers were as follows: Connective tissue growth factor (CTGF) forward, 5'-CCAATGACAACGCCT CCTG-3' and reverse, 5'-TGGTGCAGCCAGAAAGCTC-3'; cysteine-rich angiogenic inducer 61 (Cyr61) forward, 5'-AGC CTCGCATCCTATACAACC-3' and reverse, 5'-TTCTTTCAC AAGGCGGCACTC-3'; YAP forward, 5'-CAGGAATTATTT CGGCAGGA-3' and reverse, 5'-CATCCTGCTCCAGTGTAG GC-3'; and GAPDH forward, 5'-GTCTCCTCTGACTTCAAC AGCG-3' and reverse, 5'-ACCACCCTGTTGCTGTAGCCA A-3'.

Dual-luciferase reporter assays. A 375 cells with depleted YAP and/or USP22 were co-transfected with 5X UAS-luciferase reporter (cat. no. 46756), Gal4-TEAD4 (cat. no. 24640) (both Addgene, Inc.) and pRL Renilla luciferase control reporter (cat. no. E2261; Promega Corporation) using Lipofectamine 3000 . After $36 \mathrm{~h}$ of transfection, luciferase activity was measured using the Dual-Glo Luciferase Assay kit (cat. no. E2920; Promega Corporation) following the manufacturer's instructions. All luciferase activities were normalized to Renilla luciferase activity.

Cell proliferation assay. A375 cells transfected with shUSP22 and/or shYAP ( $1 \times 10^{4}$ per well) were seeded in 6-well plates in triplicates and counted manually using a light microscope (magnification, x20) every day for 5 days. Data are shown as mean cell number derived from three biological replicates.

Cell viability assay. A375 and A2058 cells, either ectopically expressing USP22 or YAP, or depleted of USP22 or YAP (2,000-3,000 per well) were seeded in 96-well plates in triplicates for $24 \mathrm{~h}$ and then treated with $1 \mu \mathrm{M}$ vemurafenib at $37^{\circ} \mathrm{C}$ for $24 \mathrm{~h}$. The viable cells were determined using CellTiter-Glo luminescent cell viability reagent according to the manufacturer's instructions (cat. no. G7570; Promega Corporation). Briefly, the aforementioned cells were incubated with CellTiter-Glo solution at room temperature for $10 \mathrm{~min}$. The luminescent signal was detected by GloMax Microplate Luminometer (cat. no. GM2000; Promega Corporation). 
Data are shown as percentage of the control cells from three biological replicates.

Mouse tumor xenograft assay. All animal experiments were performed under a protocol approved by the Institutional Animal Care and Use Committee in Changxing People's Hospital. All mice were purchased from the Model Animal Research Center of Nanjing University (Nanjing, China) and housed in a room with conditions of $22^{\circ} \mathrm{C}, 50-60 \%$ humidity and a 12-h light/12-h dark cycle. Water and food were always accessible and were checked every day. A375 cells depleted of USP22 or USP22/YAP (2x10\% /each site) were resuspended in $100 \mu \mathrm{l}$ PBS and mixed with Matrigel (cat. no. 354234; 1:1; Corning, Inc.), and were injected subcutaneously into the two front flanks of 5-week-old immunodeficient NOD-SCID male mice with a body weight of 20-22 g. In total, 15 mice were used, with 5 mice in each group, including group I (shGFP; negative control), group II (shUSP22) and group III (shUSP22+shYAP). Tumor size was measured every 3 days and tumor volumes were calculated using the following equation: $\mathrm{L}^{\mathrm{x}} \mathrm{W}^{2} \mathrm{x} 0.52$ (where $\mathrm{L}$ is length and $\mathrm{W}$ is width). At the endpoint, the mice were euthanized with $\mathrm{CO}_{2}$ in the chamber for 5 min with a displacement rate at $40 \%$ of the chamber volume per min, followed by decapitation to confirm death before being placed in the freezer.

Statistical analysis. The RT-PCR assays, luciferase reporter assays, cell proliferation and cell viability assays were performed three times. The data are shown as mean \pm standard deviation. $\mathrm{P}<0.05$ was considered to indicate a statistically significant difference between groups, which was analyzed by one-way ANOVA followed by Tukey's post hoc test for multiple comparisons or Dunnett's test for comparisons against a single control, using GraphPad Prism 8 (GraphPad Software, Inc.). In total, 90 melanoma tissue samples were used in the IHC staining assay and data are shown as the relative percentage of cases containing different staining intensities. $\mathrm{P}<0.05$ was considered to indicate significant differences, as analyzed by Fisher's exact test using GraphPad Prism 8. For the xenograft assay, the data are shown as mean \pm standard error of the mean.

\section{Results}

Expression of USP22 and YAP is associated in melanoma. USP22 is frequently overexpressed and associated with poor prognosis in various human cancer types (33-36). Notably, metastatic melanoma displays much higher expression of USP22 compared with the primary tumor, indicating it may play an important role in melanoma progression (37). Given that the protein levels of YAP are also increased in most melanomas (38), the present study investigated whether there was a possible link between USP22 and YAP. First, the protein levels were analyzed in melanoma cell lines. Compared with NHEM, all melanoma cell lines examined exhibited higher expression of USP22 and YAP. Notably, cells with higher protein levels of USP22 also displayed higher expression of YAP (Fig. 1A). Consistent with this result, IHC staining showed that both USP22 and YAP were highly expressed in $~ 50 \%$ of patient samples. Moreover, high expression of USP22 was associated with high expression of YAP (Fig. 1B and C; P<0.0001). These results suggested that elevated expression of USP22 and YAP coexisted in melanoma.

USP22 interacts with and deubiquitinates YAP. As a DUB, USP22 interacts with its substrates to remove the ubiquitin moieties (39). Having demonstrated crosstalk between USP22 and YAP, it was investigated whether USP22 functioned as a YAP deubiquitinase. Using IP assays, USP22 interaction with YAP at the endogenous level in A375 melanoma cells was observed (Fig. 2A and B). Consistently, knockdown of USP22 by shRNA in A375 cells markedly increased YAP ubiquitination (Fig. 2C). On the other hand, overexpression of USP22-WT, but not the enzymatic-dead form USP22-C185S, decreased YAP ubiquitination (Fig. 2D). These results suggested that USP22 was a deubiquitinase of YAP, which decreased YAP ubiquitination in melanoma cells.

USP22 stabilizes YAP protein. For most of its substrates, USP22 increases the protein stability by antagonizing ubiquitination-mediated protein degradation (40). To determine the important role of USP22 in governing YAP abundance, USP22 was overexpressed in A375 cells and it was found that YAP expression was elevated in a USP22 dose-dependent manner (Fig. 3A). By contrast, knockdown of USP22 by shRNA decreased YAP protein levels, but not mRNA levels (Fig. 3B and C; P>0.05). Consistently, knockdown of USP22 shortened the half-life of YAP protein in a cycloheximide chase assay (Fig. 3D). These results supported the notion that USP22 functions as a deubiquitinase to stabilize YAP protein.

USP22 promotes melanoma mainly through YAP. As a transcriptional activator, YAP exerts its oncogenic functions by promoting the transcription of downstream target genes, such as CTGF and Cyr61 (41). To assess the effect of USP22 on YAP transactivation, luciferase assays were performed using a YAP-responsive luciferase reporter system (42), and it was found that the reporter was significantly suppressed in USP22-knockdown cells (Fig. 4A and B; $\mathrm{P}<0.01$ ). Moreover, the reporter activity was similar in cells depleted with USP22 alone or both USP22 and YAP (Fig. 4A and B; P>0.05). Consistently, USP22-knockdown significantly suppressed the mRNA levels of YAP downstream target genes (Fig. 4C; $\mathrm{P}<0.01$ ), including CTGF and Cyr61, while there was no further reduction when combined with YAP depletion (Fig. 4C; P>0.05). These results indicated that USP22 controlled YAP-dependent transcription. The biological function of USP22-mediated stabilization of YAP in melanoma was investigated. It was found that silencing USP22 in A375 cells significantly suppressed cell proliferation (Fig. 4D; P<0.01). Moreover, there was no synergistic effect on cell proliferation when both USP22 and YAP were depleted (Fig. 4D; P>0.05), suggesting that USP22 promoted cell proliferation largely through YAP. To obtain in vivo evidence supporting this notion, mouse xenograft experiments were performed using A375 cells. Notably, depletion of USP22 alone displayed similar tumor inhibition effects as depletion of both USP22 and YAP (Fig. 4E and F). Together, these results suggested that YAP was a major downstream effector mediating the oncogenic function of USP22 in melanoma progression. 
A

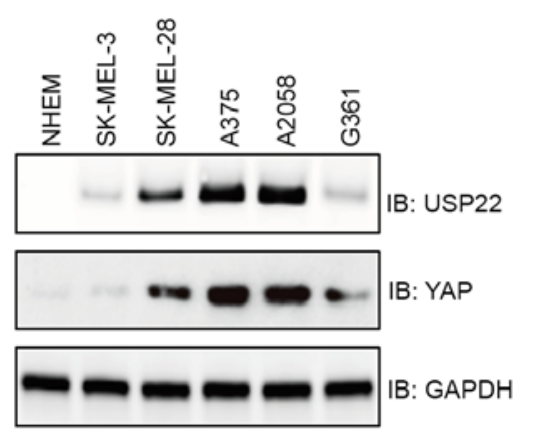

B

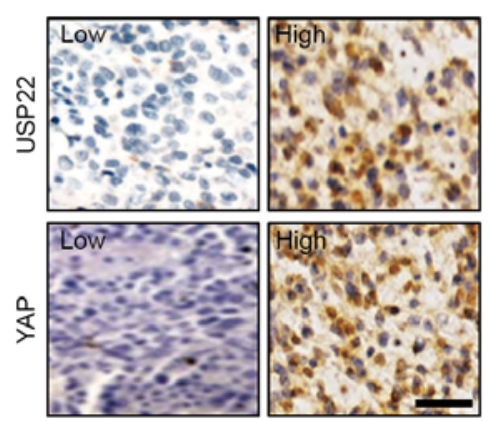

C

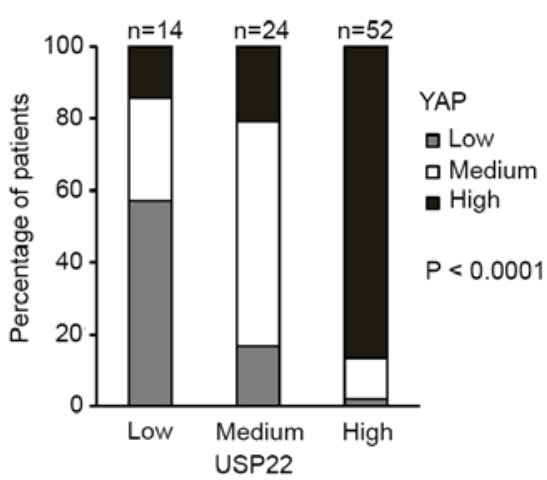

Figure 1. Elevated expression of USP22 and YAP in melanoma. (A) Analysis of USP22 and YAP expression in melanoma cell lines using western blotting. (B) Representative images of immunohistochemical staining for the expression of USP22 and YAP in tissues from patients with melanoma. Since the staining intensity between low and high was considered as medium, the range of medium is relatively wide, and no representative image was provided. Scale bar, $50 \mu \mathrm{M}$. (C) Statistical analysis of the protein levels of USP22 and YAP in melanoma samples. P $<0.0001$ indicates comparison among three groups with low, medium and high expression of USP22. YAP, yes-associated protein; USP22, ubiquitin-specific peptidase 22; IB, immunoblot.

A

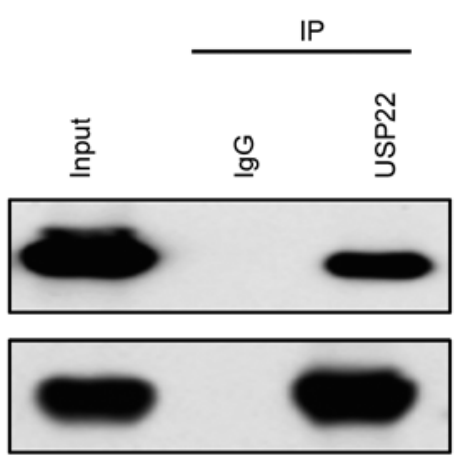

C

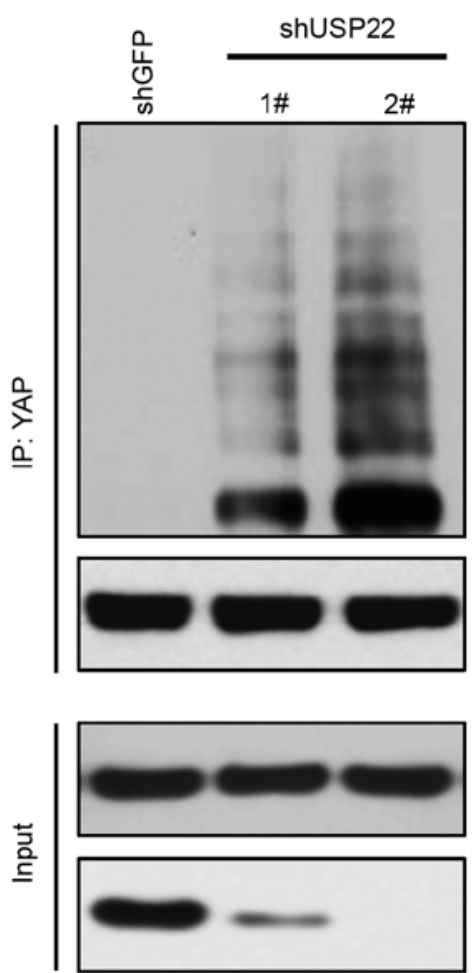

B

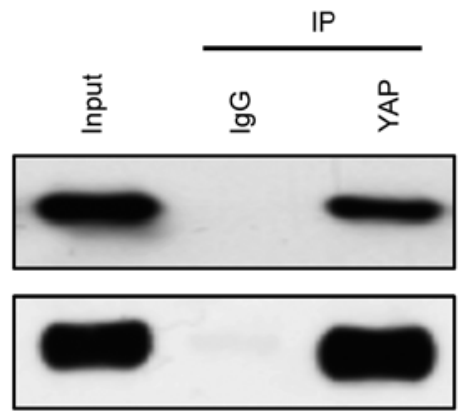

IB: USP22

IB: YAP

IB: USP22

IB: Ub

D

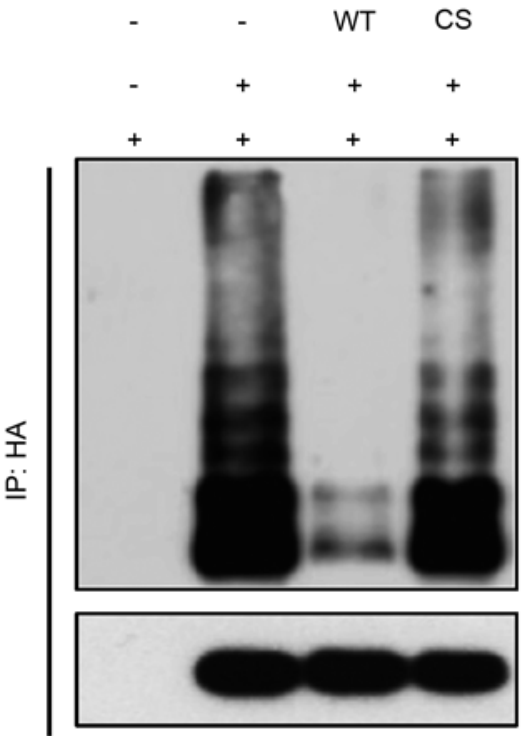

Flag-USP22

HA-YAP

Myc-Ub

IB: Myc

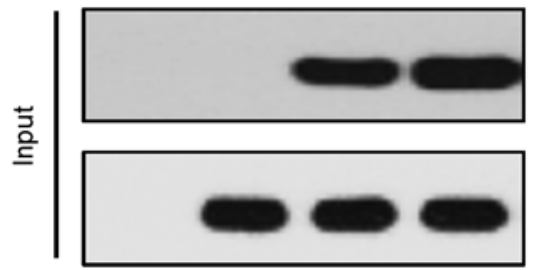

IB: HA

IB: Flag

IB: HA

Figure 2. USP22 interacts with and deubiquitinates YAP. Examination of USP22 and YAP interaction by (A) IP assays and (B) western blotting. (C) USP22-depleted A375 cells were subjected to ubiquitination assay and western blotting. (D) Western blot analysis of YAP ubiquitination derived from A375 cells transfected with indicated constructs. YAP, yes-associated protein; USP22, ubiquitin-specific peptidase 22; IP, immunoprecipitation; WT, wild-type; CS, USP22-C185S; Ub, ubiquitin; IB, immunoblot; HA, hemagglutinin. 
A

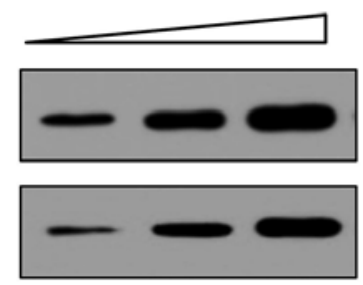

Flag-USP22

IB: YAP

IB: Flag

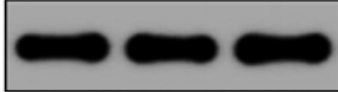

IB: GAPDH
B

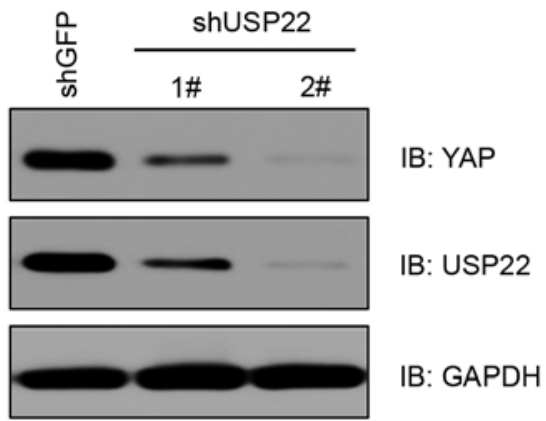

C

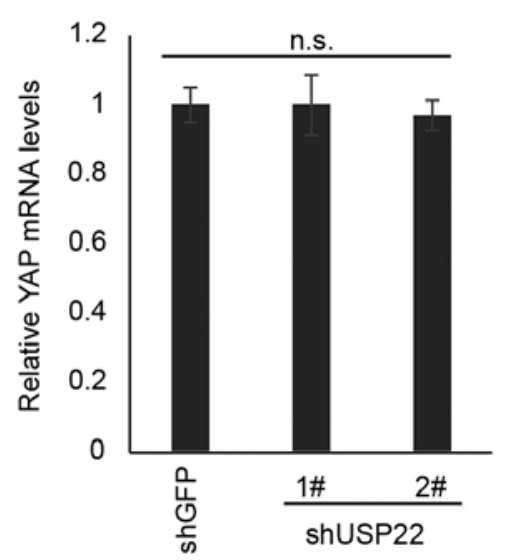

D

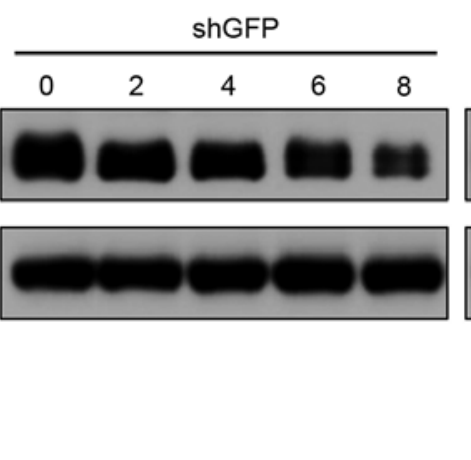

$\mathrm{CHX}(\mathrm{h})$

IB: YAP

IB: GAPDH

Figure 3. USP22 enhances YAP protein stability. (A) A375 cells were transfected different doses of USP22 construct for $48 \mathrm{~h}$ before being subjected to western blot for analysis of YAP expression. A375 cells were infected with USP22 shRNA virus and selected with puromycin for $72 \mathrm{~h}$. Cells were harvested and subjected to (B) western blot and (C) reverse transcription-quantitative PCR for analysis of YAP protein and mRNA levels, respectively. (D) Half-life of YAP was examined using cycloheximide-chase assays. n.s., non-significant vs. shGFP. YAP, yes-associated protein; USP22, ubiquitin-specific peptidase 22; sh, short hairpin; CHX, cycloheximide; IB, immunoblot.
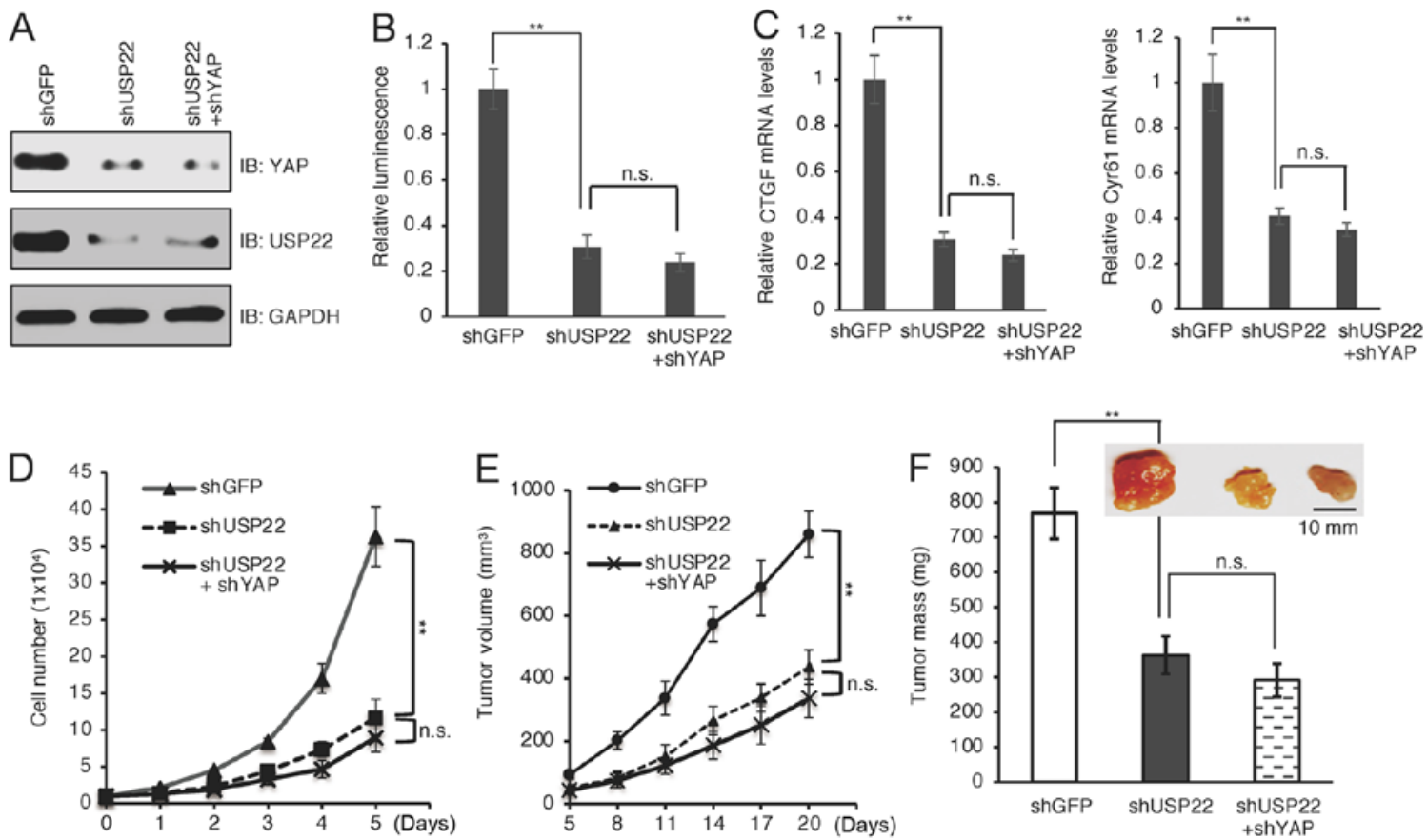

Figure 4. Depletion of USP22 suppresses YAP-dependent transcription, cell proliferation and tumor growth. (A) A375 cells were infected with YAP and/or USP shRNA and subjected to western blotting. (B) YAP- and/or USP22-depleted A375 cells were transfected with a luciferase reporter system and subjected to luciferase activity analysis. (C) Reverse transcription PCR analysis of mRNA levels of YAP downstream targets, including CTGF and Cyr61 in A375 cells depleted of USP22 and/or YAP. (D) Cell proliferation of indicated cells. (E) USP22-depleted A375 cells were used for mouse xenograft assays. (F) Representative subcutaneous tumors were shown and statistical analysis of tumor weight. Scale bar, $10 \mathrm{~mm} .{ }^{* *} \mathrm{P}<0.01$ shUSP22 vs. shGFP. YAP, yes-associated protein; USP22, ubiquitin-specific peptidase 22; sh, short hairpin; n.s., non-significant; CTGF, connective tissue growth factor; Cyr61, cysteine-rich angiogenic inducer 61 ; IB, immunoblot. 

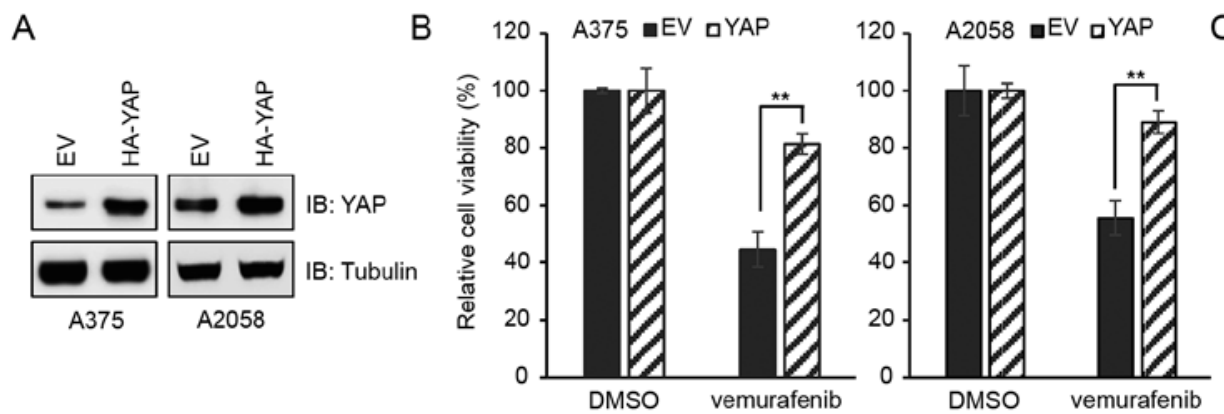

C

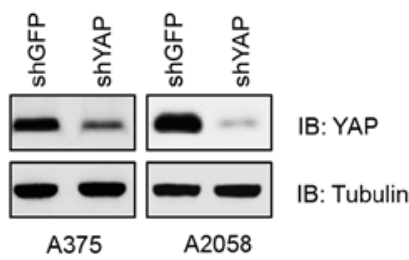

D

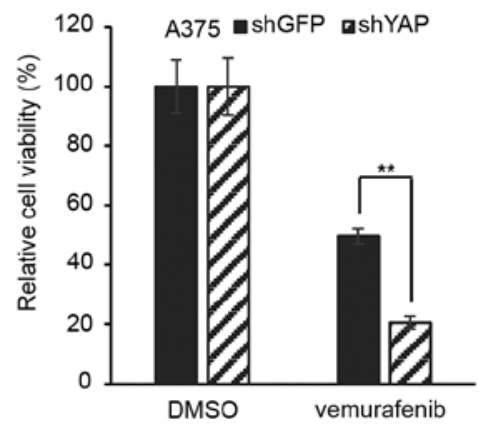

F

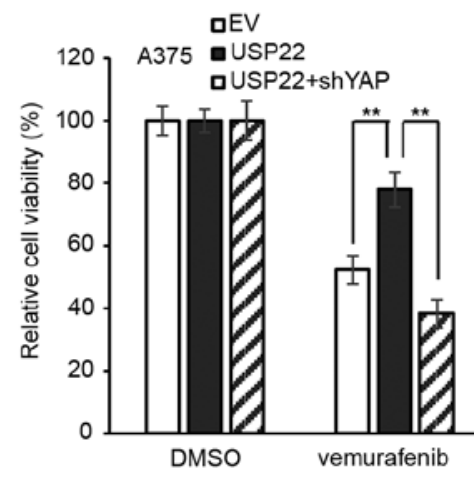

$\mathrm{H}$

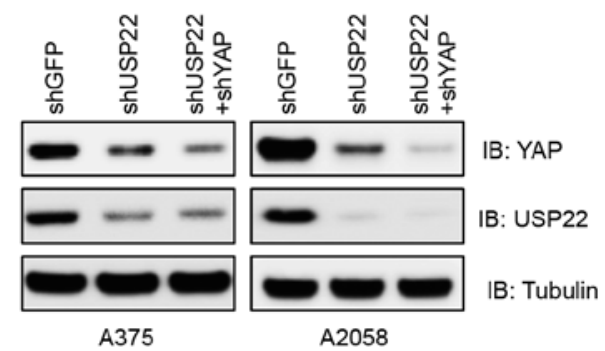

I
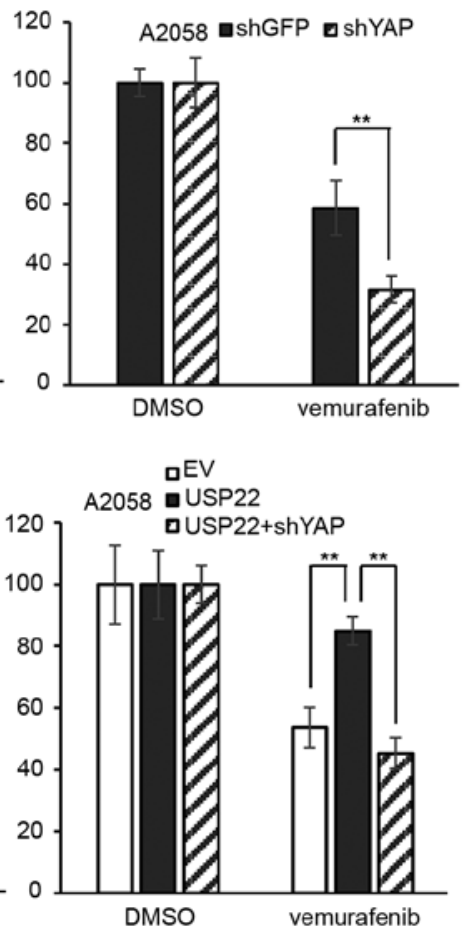

E

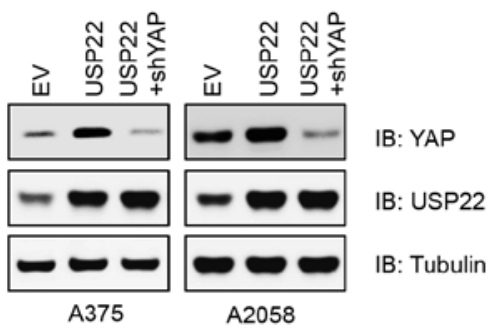

G
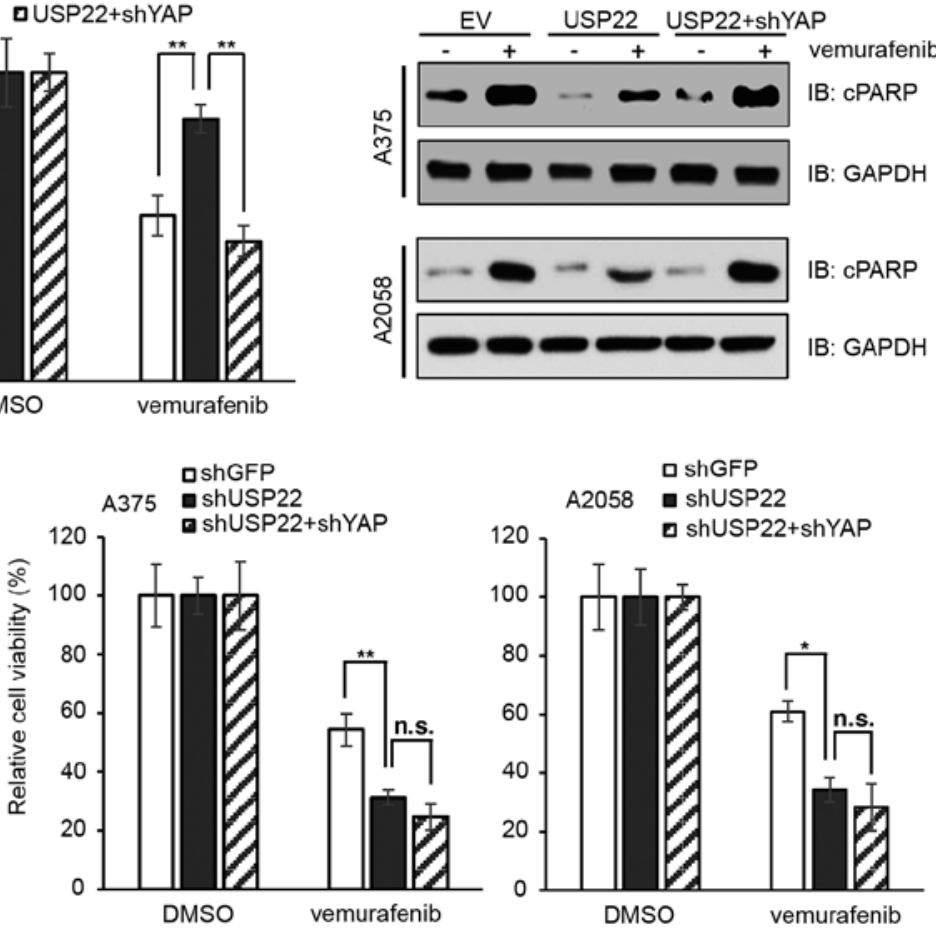

Figure 5. USP22 dictates cellular sensitivity to the BRAF inhibitor vemurafenib. (A) Western blot analysis of YAP expression in A375 and A2058 cells transfected with EV or HA-YAP construct. (B) Cell survival of A375 and A2058 cells generated in (A). (C) Western blot analysis of YAP expression in A375 and A2058 cells depleted of YAP. (D) Cell survival of A375 and A2058 cells generated in (C). Cells were treated with $1 \mu \mathrm{M}$ vemurafenib for $24 \mathrm{~h}$. (E) Western blot analysis of YAP and USP22 expression in A375 and A2058 cells expressing EV or USP22 and/or YAP shRNA. (F) Cell survival of A375 and A2058 cells generated in (E). (G) Western blot analysis of A375 and A2058 cells generated in (E) that were treated with $1 \mu \mathrm{M}$ vemurafenib for $24 \mathrm{~h}$. (H) Western blot analysis of YAP and USP22 expression in A375 and A2058 cells depleted of USP22 and/or YAP. (I) Cell survival of A375 and A2058 cells generated in $(\mathrm{H}){ }^{* *} \mathrm{P}<0.01$ vs. EV, USP22 or shGFP. YAP, yes-associated protein; USP22, ubiquitin-specific peptidase 22; sh, short hairpin; n.s., non-significant; cPARP, cleaved PARP; EV, empty vector; IB, immunoblot.

USP22 confers resistance to vemurafenib in melanoma cells. Different cell lines were generated to test the contribution of USP22 and YAP in vemurafenib resistance. These included cells transfected with EV (pcDNA3.1) or HA-YAP (Fig. 5A), cells infected with shGFP or shYAP virus (Fig. 5C), cells expressing EV (pcDNA3.1) or USP22 and/or shYAP (Fig. 5E) and cells infected with shGFP, shUSP 22 or shUSP $22+$ shYAP (Fig. 5H). Consistent with earlier reports where the elevation of YAP expression contributed to resistance to BRAF-targeted therapy in melanoma $(24,25)$, the present study reported that 
compared with ectopic expression of EV, ectopic expression of YAP promoted cell survival following vemurafenib treatment (Fig. 5B; P<0.01). By contrast, depletion of YAP decreased viability when treated with vemurafenib (Fig. 5D; $\mathrm{P}<0.01$ ). Notably, compared with ectopic expression of EV (pcDNA3.1), ectopic expression of USP22 suppressed vemurafenib-induced cell death, which could be reversed by $Y A P$-silencing (Fig. 5F; $\mathrm{P}<0.01$ ). Moreover, upon vemurafenib treatment, the protein levels of apoptosis marker cPARP were lower in USP22-overexpressed cells compared with EV-treated cells (Fig. 5G). Furthermore, cells depleted of USP22 or depleted of USP22 plus YAP exhibited similar sensitivity to vemurafenib (Fig. 5I). These data suggested that USP22 dictated cell sensitivity to the BRAF inhibitor vemurafenib via YAP.

\section{Discussion}

Ubiquitination can be reversed by deubiquitinating enzymes that remove ubiquitin from targets to regulate protein activity and stability. In humans, there are 100 DUBs, which belong to five sub-families: USPs (the largest subfamily), ovarian tumor proteases, ubiquitin carboxy-terminal hydrolases, Machado-Joseph disease protein domain proteases and JAB1/MPN/Mov34 metalloenzyme domain metalloproteases (43). As the antagonist of ubiquitination, DUBs play important roles in numerous cellular processes, including transcription, DNA repair, protein degradation and signaling transduction. DUBs can be an oncogenes or tumor suppressors depending on their substrates or context. Dysregulation of DUBs is associated with various human diseases ranging from cancer to neurological disorders $(44,45)$.

USP22 is a member of the USP family, which is evolutionarily conserved from yeast to humans; it contains a zinc finger domain at the $\mathrm{N}$-terminus that binds other three components to form the deubiquitinating module. The catalytic domain is located at the C-terminus (46). USP22 was initially reported to promote deubiquitylation of histones $\mathrm{H} 2 \mathrm{~A}$ and $\mathrm{H} 2 \mathrm{~B}$, leading to transcription activation (47). USP22 also deubiquitinates non-histone proteins, including telomeric repeat-binding factor 1 (TRF1), sirtuin 1 (SIRT1), cyclin B1 and others (40), leading to protein stabilization by preventing proteasome-mediated degradation. For example, USP22 promotes TRF1 deubiquitylation to enhance TRF1 protein stability and maintain telomere integrity (48). In the present study, it was revealed that USP22 specifically interacted with and deubiquitinated YAP. Moreover, depletion of USP22 decreased YAP protein expression. As a result, USP22-knockdown significantly suppressed melanoma cell proliferation and tumor growth in a xenograft mouse model, which was similar to YAP-knockdown. Therefore, the current findings indicated that YAP may be a critical downstream mediator of USP22 in tumorigenesis. However, the substrate and ubiquitin chain specificity for USP22 is unknown, which will be an interesting research topic in the future. The present identification of YAP as its novel substrate might provide insight into this puzzle.

USP22 is largely considered as an oncoprotein, which is frequently overexpressed in several types of cancer, including lung cancer, pancreatic cancer, salivary adenoid cystic carcinoma, prostate cancer and liver cancer, and associated with poor overall survival (49-52). It was reported that USP22 promoted lung cancer by regulating pathways of ubiquitination and immunosuppression (33). USP22 also deubiquitinated and stabilized CDC274 (PD-L1) to decrease the efficacy of CD274-targeted immunotherapy in mice (53). Furthermore, USP22 facilitated prostate cancer progression through enhancing androgen receptor- and Myc-driven oncogenic signaling pathways (34). Similarly, overexpression of YAP was associated with poor patient survival in numerous types of cancer, including colon cancer, lymph node metastatic melanoma and pancreatic cancer (54-56). Moreover, the Human Protein Atlas project also showed that higher expression of USP22 and YAP is associated with decreased survival in patients with melanoma (57). The present study also observed that USP22 was highly expressed in patients with melanoma via unknown molecular mechanisms. Moreover, higher USP22 expression was positively associated with higher YAP expression in melanoma cell lines and patient samples. The current findings provide a possible molecular mechanism for aberrant YAP expression in melanoma. USP22 has been reported to be regulated at both transcriptional and post-translational levels. For example, SP1 and protein kinase A/cAMP response element-binding protein could bind to the USP22 promoter to suppress or promote USP22 transcription, respectively $(58,59)$. Phosphorylation of T147 and S237 by cyclin-dependent kinase 1 or acetylation of K129 leads to enhanced USP22 deubiquitinase activity $(60,61)$. Moreover, anaphase-promoting complex cell division cycle protein $20\left(\mathrm{APC}^{\mathrm{CDC} 20}\right)$, an E3 ubiquitin ligase, promotes USP22 degradation in a cell cycle-dependent manner (61). It will be interesting to determine whether $\mathrm{APC}^{\mathrm{CDC} 20}$ or other mechanisms contribute to USP22 and YAP overexpression in melanoma.

Another notable finding in the current study was that overexpression of USP22 resulted in resistance to the BRAF inhibitor vemurafenib. One of the milestones in the melanoma research field was the discovery of the BRAF-V600E mutation, leading to the rapid development of targeted therapies to improve overall survival rate for patients with melanoma $(14,62)$. However, acquired resistance to these targeted therapies also emerged due to different mechanisms. For example, additional genetic alteration was considered the main mechanism, which led to the recovery of the MAPK pathway and activation of $\mathrm{PI} 3 \mathrm{~K} / \mathrm{Akt} / \mathrm{mTOR}$ signaling (63). Notably, in a genetic screen that silenced $>5,000$ targets, YAP stood out to be a critical determinant of BRAF inhibitor resistance. Deletion of YAP resulted in the best response to vemurafenib treatment in various types of cancer cells harboring the BRAF-V600E mutation (25). Consistently, the present study determined that USP22 is a key regulator of YAP expression and BRAF inhibitor resistance in melanoma.

Overall, the current study provided evidence showing that USP22 functions as the deubiquitinase to govern YAP ubiquitination and protein stability. Overexpression of USP22 lead to elevation of YAP protein levels and subsequent resistance to vemurafenib in melanoma. Therefore, the present study provided a molecular mechanism and rationale for combined inhibition of USP22/YAP and BRAF as an option for melanoma treatment. However, a limitation of the current study was that most experiments were performed using melanoma cells. Further studies using animal models, such as USP22-knockout 
mice, are required to demonstrate the critical role of the USP22/YAP axis in melanoma and BRAF inhibitor resistance.

\section{Acknowledgements}

Not applicable.

\section{Funding}

No funding was received.

\section{Availability of data and materials}

All data generated or analyzed during this study are included in this published article.

\section{Authors' contributions}

YW and ZJ performed the experiments. YW and JL analyzed the data, confirmed its authenticity and wrote the manuscript. All authors read and approved the final manuscript.

\section{Ethics approval and consent to participate}

The present study was approved by the Institutional Research Ethics Committee at Changxing People's Hospital (Huzhou, China). Written informed consent was obtained from patients and/or their guardians.

\section{Patient consent for publication}

Not applicable.

\section{Competing interests}

The authors declare that they have no competing interests.

\section{References}

1. Shain AH and Bastian BC: From melanocytes to melanomas. Nat Rev Cancer 16: 345-358, 2016.

2. Davis LE, Shalin SC and Tackett AJ: Current state of melanoma diagnosis and treatment. Cancer Biol Ther 20: 1366-1379, 2019.

3. Siegel RL, Miller KD and Jemal A: Cancer statistics, 2020. CA Cancer J Clin 70: 7-30, 2020

4. Rodriguez-Cerdeira C, Carnero Gregorio M, Lopez-Barcenas A, Sánchez-Blanco E, Sánchez-Blanco B, Fabbrocini G, Bardhi B, Sinani A and Guzman RA: Advances in immunotherapy for melanoma: A comprehensive review. Mediators Inflamm 2017: 3264217, 2017.

5. Domingues B, Lopes JM, Soares P and Populo H: Melanoma treatment in review. Immunotargets Ther 7: 35-49, 2018.

6. Weiss SA, Wolchok JD and Sznol M: Immunotherapy of melanoma: Facts and hopes. Clin Cancer Res 25: 5191-5201, 2019.

7. Bhandaru M and Rotte A: Monoclonal antibodies for the treatment of melanoma: Present and future strategies. Methods Mol Biol 1904: 83-108, 2019.

8. Kozar I, Margue C, Rothengatter S, Haan C and Kreis S: Many ways to resistance: How melanoma cells evade targeted therapies. Biochim Biophys Acta Rev Cancer 1871: 313-322, 2019.

9. Lugowska I, Teterycz P and Rutkowski P: Immunotherapy of melanoma. Contemp Oncol (Pozn) 22: 61-67, 2018.

10. Paluncic J, Kovacevic Z, Jansson PJ, Kalinowski D, Merlot AM, Huang ML, Lok HC, Sahni S, Lane DJ and Richardson DR: Roads to melanoma: Key pathways and emerging players in melanoma progression and oncogenic signaling. Biochim Biophys Acta 1863: 770-784, 2016.
11. Haluska FG, Tsao H, Wu H, Haluska FS, Lazar A and Goel V: Genetic alterations in signaling pathways in melanoma. Clin Cancer Res 12: 2301s-2307s, 2006.

12. Wang Y and Chen Z: Mutation detection and molecular targeted tumor therapies. STEMedicine 1: e11, 2020.

13. Mehnert JM and Kluger HM: Driver mutations in melanoma: Lessons learned from bench-to-bedside studies. Curr Oncol Rep 14: 449-457, 2012.

14. Savoia P, Fava P, Casoni F and Cremona O: Targeting the ERK signaling pathway in melanoma. Int J Mol Sci 20: 1483, 2019.

15. Fujimura T, Fujisawa Y, Kambayashi Y and Aiba S: Significance of BRAF kinase inhibitors for melanoma treatment: From bench to bedside. Cancers (Basel) 11: 1342, 2019.

16. Luebker SA and Koepsell SA: Diverse mechanisms of BRAF inhibitor resistance in melanoma identified in clinical and preclinical studies. Front Oncol 9: 268, 2019.

17. Johnson DB, Menzies AM, Zimmer L, Eroglu Z, Ye F, Zhao S, Rizos H, Sucker A, Scolyer RA, Gutzmer R, et al: Acquired BRAF inhibitor resistance: A multicenter meta-analysis of the spectrum and frequencies, clinical behaviour, and phenotypic associations of resistance mechanisms. Eur J Cancer 51: 2792-2799, 2015.

18. Ma S, Meng Z, Chen R and Guan KL: The hippo pathway: Biology and pathophysiology. Annu Rev Biochem 88: 577-604, 2019.

19. Kim MK, Jang JW and Bae SC: DNA binding partners of YAP/TAZ. BMB Rep 51: 126-133, 2018.

20. Moroishi T, Hansen CG and Guan KL: The emerging roles of YAP and TAZ in cancer. Nat Rev Cancer 15: 73-79, 2015.

21. Zanconato F, Cordenonsi $M$ and Piccolo S: YAP/TAZ at the roots of cancer. Cancer Cell 29: 783-803, 2016.

22. Xiong H, Yu Q, Gong Y, Chen W, Tong Y, Wang Y, Xu H and Shi Y: Yes-Associated protein (YAP) promotes tumorigenesis in melanoma cells through stimulation of low-density lipoprotein receptor-related protein 1 (LRP1). Sci Rep 7: 15528, 2017.

23. Nallet-Staub F, Marsaud V, Li L, Gilbert C, Dodier S, Bataille V, Sudol M, Herlyn M and Mauviel A: Pro-invasive activity of the Hippo pathway effectors YAP and TAZ in cutaneous melanoma. J Invest Dermatol 134: 123-132, 2014.

24. Fisher ML, Grun D, Adhikary G, Xu W and Eckert RL: Inhibition of YAP function overcomes BRAF inhibitor resistance in melanoma cancer stem cells. Oncotarget 8: 110257-110272, 2017.

25. Lin L, Sabnis AJ, Chan E, Olivas V, Cade L, Pazarentzos E, Asthana S, Neel D, Yan JJ, Lu X, et al: The Hippo effector YAP promotes resistance to RAF- and MEK-targeted cancer therapies. Nat Genet 47: 250-256, 2015.

26. Meng Z, Moroishi T and Guan KL: Mechanisms of Hippo pathway regulation. Genes Dev 30: 1-17, 2016.

27. Yan F, Qian M, He Q, Zhu H and Yang B: The posttranslational modifications of Hippo-YAP pathway in cancer. Biochim Biophys Acta Gen Subj 1864: 129397, 2020.

28. Zhao B, Li L, Tumaneng K, Wang CY and Guan KL: A coordinated phosphorylation by Lats and CK1 regulates YAP stability through SCF (beta-TRCP). Genes Dev 24: 72-85, 2010.

29. Tu K, Yang W, Li C, Zheng X, Lu Z, Guo C, Yao Y and Liu Q: Fbxw7 is an independent prognostic marker and induces apoptosis and growth arrest by regulating YAP abundance in hepatocellular carcinoma. Mol Cancer 13: 110, 2014.

30. Yao F, Zhou Z, Kim J, Hang Q, Xiao Z, Ton BN, Chang L, Liu N, Zeng L, Wang W, et al: SKP2- and OTUD1-regulated non-proteolytic ubiquitination of YAP promotes YAP nuclear localization and activity. Nat Commun 9: 2269, 2018.

31. Tiscornia G, Singer O and Verma IM: Production and purification of lentiviral vectors. Nat Protoc 1: 241-245, 2006.

32. Livak KJ and Schmittgen TD: Analysis of relative gene expression data using real-time quantitative PCR and the 2(-Delta Delta $\mathrm{C}(\mathrm{T}))$ method. Methods 25: 402-408, 2001.

33. Han B, Sun Y, Yang D, Zhang H, Mo S, Chen X, Lu H, Mao X and $\mathrm{Hu}$ J: USP22 promotes development of lung adenocarcinoma through ubiquitination and immunosuppression. Aging (Albany NY) 12: 6990-7005, 2020.

34. Schrecengost RS, Dean JL, Goodwin JF, Schiewer MJ, Urban MW, Stanek TJ, Sussman RT, Hicks JL, Birbe RC, Draganova-Tacheva RA, et al: USP22 regulates oncogenic signaling pathways to drive lethal cancer progression. Cancer Res 74: 272-286, 2014.

35. Yang X, Zang H, Luo Y, Wu J, Fang Z, Zhu W and Li Y: High expression of USP22 predicts poor prognosis and advanced clinicopathological features in solid tumors: A meta-analysis. Onco Targets Ther 11: 3035-3046, 2018. 
36. McCann JJ, Vasilevskaya IA, Poudel Neupane N, Shafi AA, McNair C, Dylgjeri E, Mandigo AC, Schiewer MJ, Schrecengost RS, Gallagher P, et al: USP22 functions as an oncogenic driver in prostate cancer by regulating cell proliferation and DNA repair. Cancer Res 80: 430-443, 2020.

37. Luise C, Capra M, Donzelli M, Mazzarol G, Jodice MG, Nuciforo P, Viale G, Di Fiore PP and Confalonieri S: An atlas of altered expression of deubiquitinating enzymes in human cancer. PLoS One 6: e15891, 2011.

38. Zhang X, Tang JZ, Vergara IA, Zhang Y, Szeto P, Yang L, Mintoff C, Colebatch A, McIntosh L, Mitchell KA, et al: Somatic hypermutation of the YAP oncogene in a human cutaneous melanoma. Mol Cancer Res 17: 1435-1449, 2019.

39. Komander D, Clague MJ and Urbe S: Breaking the chains: Structure and function of the deubiquitinases. Nat Rev Mol Cel Biol 10: 550-563, 2009.

40. Melo-Cardenas J, Zhang Y, Zhang DD and Fang D: Ubiquitin-specific peptidase 22 functions and its involvement in disease. Oncotarget 7: 44848-44856, 2016.

41. Yu FX, Zhao B, Panupinthu N, Jewell JL, Lian I, Wang LH, Zhao J, Yuan H, Tumaneng K, Li H, et al: Regulation of the Hippo-YAP pathway by G-protein-coupled receptor signaling. Cell 150: 780-791, 2012.

42. Zhao B, Wei X, Li W, Udan RS, Yang Q, Kim J, Xie J, Ikenoue T, $\mathrm{Yu} \mathrm{J}, \mathrm{Li} \mathrm{L}$, et al: Inactivation of YAP oncoprotein by the Hippo pathway is involved in cell contact inhibition and tissue growth control. Genes Dev 21: 2747-2761, 2007.

43. Nijman SM, Luna-Vargas MP, Velds A, Brummelkamp TR, Dirac AM, Sixma TK and Bernards R: A genomic and functiona inventory of deubiquitinating enzymes. Cell 123: 773-786, 2005.

44. Reyes-Turcu FE, Ventii KH and Wilkinson KD: Regulation and cellular roles of ubiquitin-specific deubiquitinating enzymes Annu Rev Biochem 78: 363-397, 2009.

45. Liu L, Yin S, Brobbey C and Gan W: Ubiquitination in cancer stem cell: Roles and targeted cancer therapy. STEMedicine 1: e37, 2020.

46. Samara NL, Datta AB, Berndsen CE, Zhang X, Yao T, Cohen RE and Wolberger C: Structural insights into the assembly and function of the SAGA deubiquitinating module. Science 328 : 1025-1029, 2010

47. Zhao Y, Lang G, Ito S, Bonnet J, Metzger E, Sawatsubashi S, Suzuki E, Le Guezennec X, Stunnenberg HG, Krasnov A, et al: A TFTC/STAGA module mediates histone $\mathrm{H} 2 \mathrm{~A}$ and $\mathrm{H} 2 \mathrm{~B}$ deubiquitination, coactivates nuclear receptors, and counteracts heterochromatin silencing. Mol Cell 29: 92-101, 2008.

48. Atanassov BS, Evrard YA, Multani AS, Zhang Z, Tora L, Devys D, Chang S and Dent SY: Gen5 and SAGA regulate shelterin protein turnover and telomere maintenance. Mol Cell 35 352-364, 2009

49. Ning J, Zhang J, Liu W, Lang Y, Xue Y and Xu S: Overexpression of ubiquitin-specific protease 22 predicts poor survival in patients with early-stage non-small cell lung cancer. Eur J Histochem 56 e46, 2012.

50. Tang B, Liang X, Tang F, Zhang J, Zeng S, Jin S, Zhou L, Kudo Y and Qi G: Expression of USP22 and Survivin is an indicator of malignant behavior in hepatocellular carcinoma. Int J Oncol 47: 2208-2216, 2015.
51. Ning Z, Wang A, Liang J, Xie Y, Liu J, Feng L, Yan Q and Wang Z: USP22 promotes the G1/S phase transition by upregulating FoxM1 expression via beta-catenin nuclear localization and is associated with poor prognosis in stage II pancreatic ductal adenocarcinoma. Int J Oncol 45: 1594-1608, 2014.

52. Dai W, Yao Y,Zhou Q and Sun CF: Ubiquitin-specific peptidase 22, a histone deubiquitinating enzyme, is a novel poor prognostic factor for salivary adenoid cystic carcinoma. PLoS One 9: e87148, 2014.

53. Huang X, Zhang Q, Lou Y, Wang J, Zhao X, Wang L, Zhang X, Li S, Zhao Y, Chen Q, et al: USP22 Deubiquitinates CD274 to suppress anticancer immunity. Cancer Immunol Res 7: 1580-1590, 2019

54. Wang L, Shi S, Guo Z, Zhang X, Han S, Yang A, Wen W and Zhu Q: Overexpression of YAP and TAZ is an independent predictor of prognosis in colorectal cancer and related to the proliferation and metastasis of colon cancer cells. PLoS One 8: e65539, 2013.

55. Lee CK, Jeong SH, Jang C, Bae H, Kim YH, Park I, Kim SK and Koh GY: Tumor metastasis to lymph nodes requires YAP-dependent metabolic adaptation. Science 363: 644-649, 2019.

56. Rozengurt E, Sinnett-Smith J and Eibl G: Yes-associated protein (YAP) in pancreatic cancer: At the epicenter of a targetable signaling network associated with patient survival. Signal Transduct Target Ther 3: 11, 2018.

57. Uhlen M, Fagerberg L, Hallstrom BM, Lindskog C, Oksvold P, Mardinoglu A, Sivertsson Å, Kampf C, Sjöstedt E, Asplund A, et al: Proteomics. Tissue-based map of the human proteome. Science 347: 1260419,2015

58. Xiong J, Che X, Li X, Yu H, Gong Z and Li W: Cloning and characterization of the human USP2 2 gene promoter. PLoS One 7 : e52716, 2012

59. Xiong J, Zhou X, Gong Z, Wang T, Zhang C, Xu X, Liu J and $\mathrm{Li}$ W: PKA/CREB regulates the constitutive promoter activity of the USP22 gene. Oncol Rep 33: 1505-1511, 2015.

60. Armour SM, Bennett EJ, Braun CR, Zhang XY, McMahon SB, Gygi SP, Harper JW and Sinclair DA: A high-confidence interaction map identifies SIRT1 as a mediator of acetylation of USP22 and the SAGA coactivator complex. Mol Cell Biol 33: 1487-1502, 2013.

61. Lin Z, Tan C, Qiu Q, Kong S, Yang H, Zhao F, Liu Z, Li J, Kong Q, Gao B, et al: Ubiquitin-specific protease 22 is a deubiquitinase of CCNB1. Cell Discov 1: 15028, 2015.

62. Davies H, Bignell GR, Cox C, Stephens P, Edkins S, Clegg S, Teague J, Woffendin H, Garnett MJ, Bottomley W, et al: Mutations of the BRAF gene in human cancer. Nature 417: 949-954, 2002.

63. Manzano JL, Layos L, Buges C, de Los Llanos Gil M, Vila L, Martínez-Balibrea E and Martínez-Cardús A: Resistant mechanisms to BRAF inhibitors in melanoma. Ann Transl Med 4: 237, 2016.

c) (7) $\Theta$ This work is licensed under a Creative Commons Attribution-NonCommercial-NoDerivatives 4.0 International (CC BY-NC-ND 4.0) License. 